\title{
Ações profiláticas relacionadas a eventos adversos em idosos internados em Unidades de Terapia Intensiva: reflexões acerca do processo de educação em saúde
}

\author{
Prophylactic actions related to adverse events in elderly people hospitalized in Intensive Care
}

Units: reflections on the health education process
Acciones profilácticas relacionadas a eventos adversos en ancianos hospitalizados en Unidades de
Cuidados Intensivos: reflexiones sobre el proceso de educación en salud

Leandro Barbosa Teixeira ORCID: https://orcid.org/0000-0002-9444-7828

Universidade Estácio de Sá, Brasil

E-mail: Lbtteixeira@hotmail.com

Allan Bruno Alves de Sousa Santos ORCID: https://orcid.org/0000-0001-6412-7164

Faculdade de Educação São Francisco, Brasil E-mail: Allan.enfer1@gmail.com

Victória Maria Pontes Martins ORCID: https://orcid.org/0000-0002-8281-0132

Centro Universitário INTA, Brasil

E-mail: victoriapontes2014@hotmail.com

Joelma Maria dos Santos da Silva Apolinário ORCID: https://orcid.org/0000-0001-9521-9432 Centro Universitário Maurício de Nassau, Brasil E-mail: jo.silva00@ hotmail.com

Moisés da Silva Rêgo

ORCID: https://orcid.org/0000-0003-4882-6832

Centro Universitário Santo Agostinho, Brasil

E-mail: moises_rego42@hotmail.com

Andressa Oliveira das Chagas Morais ORCID: https://orcid.org/0000-0001-7982-8107

Centro Universitário Santo Agostinho, Brasil

E-mail: andressaoliveiramorais@hotmail.com

Victoria Freitas de Souza Moura ORCID: https://orcid.org/0000-0001-7052-1205 Centro Universitário UniFacid, Brasil

E-mail: victoriafreitasdesousamoura@hotmail.com Bianca Marçal Kós

ORCID: https://orcid.org/0000-0001-5790-0817

Universidade Federal do Maranhão, Brasil

E-mail: bianca.mk@discente.ufma.br

Thalita Rayanne da Silva Silva ORCID: https://orcid.org/0000-0003-2239-8649 Faculdade Santa Terezinha, Brasil

E-mail: silvathalitarayanne@gmail.com

Natalia Maria Chagas Evangelista ORCID: https://orcid.org/0000-0003-0095-4106 Universidade Federal do Ceará, Brasil

E-mail: nataliaevangelista2009@hotmail.com

Raimundo Renato da Silva Neto ORCID: https://orcid.org/0000-0002-9854-7877 Universidade Ceuma, Brasil

E-mail: renatosneto@gmail.com

Aline Souza de Castro

ORCID: https://orcid.org/0000-0003-4258-2056 Uniceplac, Brasil

E-mail: alinecastro0303@gmail.com

Luiz Cláudio Galera De Oliveira Filho ORCID: https://orcid.org/0000-0003-0537-9489

Universidade Estácio de Sá, Brasil

E-mail: enfermeiro.luizgalera @gmail.com 


\author{
Ellen Amanda Silva de Santana \\ ORCID: https://orcid.org/0000-0001-6067-6131 \\ Universidade Federal de Pernambuco, Brasil \\ E-mail: amaandasiilva001@gmail.com \\ Caroline Milanez Paixão \\ ORCID: https://orcid.org/0000-0002-9669-3109 \\ Universidade Federal do Piaú, Brasil \\ E-mail: enfcarolmilanez@gmail.com \\ Luiz Paulo Ribeiro da Rocha \\ ORCID: https://orcid.org/0000-0003-1440-1502 \\ Universidade Estácio de Sá, Brasil \\ E-mail: luizrochaenf@gmail.com \\ Ludmilla Rafaela Marinho da Silva \\ ORCID: https://orcid.org/0000-0003-0025-2630 \\ Pontifícia Universidade católica de Minas Gerais, Brasil \\ E-mail: ludmillarafaela30@gmail.com \\ Jaciara Pereira de Moura \\ ORCID: https://orcid.org/0000-0003-0679-9872 \\ Centro Universitário Santo Agostinho, brasil \\ E-mail: jaciaramoura18@gmail.com \\ Maria Eduarda Ninomia Taia \\ ORCID: https://orcid.org/0000-0001-9964-9579 \\ Universidade Federal do Triângulo Mineiro, Brasil \\ E-mail: mariaeduardant2561@gmail.com \\ Luciana Carneiro de Brito Ramalho \\ ORCID: https://orcid.org/0000-0002-4258-8143 \\ Faculdade Bezerra de Araújo, Brasil \\ E-mail: luciana016@gmail.com
}

\begin{abstract}
Resumo
A relevância do estudo, aplica-se pela importância de se debater essa temática, e assim possibilitar uma reflexão crítica acerca dos métodos de educação em saúde. Objetivou-se refletir acerca das medidas profiláticas relacionadas a eventos adversos em Unidades de Tratamento Intensivo em pacientes idosos, relacionando com a importância do processo de educação em saúde. Trata-se de uma revisão narrativa da literatura, de aspecto qualitativo. A busca de dados ocorreu nas bases e bancos: Base de Dados em Enfermagem (BDENF), Literatura Latino-Americana e do Caribe em Ciências da Saúde (LILACS), Medline, e Scientific Eletronic Library Online (SciELO), identificados através da Biblioteca Virtual em Saúde (BVS) e PubMed. A pesquisa ocorreu no período de novembro de 2021 a 15 de janeiro de 2022, e foram usados os descritores "Idosos", "Prevenção", "Eventos adversos", "Unidade de Terapia Intensiva" e "Educação em saúde", estabelecidos na lista Descritores em Ciências da Saúde (DECs). Constatou-se que a ocorrência de eventos adversos está diretamente ligada a falhas na assistência e falta de conhecimento para a execução de práticas profiláticas especificas para cada complicação, sendo os principais relacionados a medicações, infecções e feridas. Conclui-se que a produção de reflexões acerca dessa temática é fundamental para despertar nos profissionais e comunidade, a importância de debater assuntos relacionados a educação em saúde, e assim melhorar os cuidados ofertados a idosos em terapia intensiva.
\end{abstract}

Palavras-chave: Idosos; Prevenção; Eventos adversos; Unidade de Terapia Intensiva; Educação em saúde.

\begin{abstract}
The relevance of the study is applied due to the importance of debating this theme, and thus enabling a critical reflection on the methods of health education. The objective was to reflect on the prophylactic measures related to adverse events in Intensive Care Units in elderly patients, relating to the importance of the health education process. This is a narrative review of the literature, with a qualitative aspect. The data search took place in the following databases: Database on Nursing (BDENF), Latin American and Caribbean Literature on Health Sciences (LILACS), Medline, and Scientific Electronic Library Online (SciELO), identified through the Virtual Library. in Health (BVS) and PubMed. The research took place from November 2021 to January 15, 2022, and the descriptors "Elderly", "Prevention", "Adverse Events", "Intensive Care Unit" and "Health education" established in the list were used. Health Science Descriptors (DECs). It was found that the occurrence of adverse events is directly linked to failures in care and lack of knowledge to carry out specific prophylactic practices for each complication, the main ones being related to medications, infections and wounds. It is concluded that the production of reflections on this theme is essential to awaken in professionals and the community the importance of debating issues related to health education, and thus improve the care offered to the elderly in intensive care.
\end{abstract}

Keywords: Elderly; Prevention; Adverse events; Intensive Care Unit; Health education.

\title{
Resumen
}

La pertinencia del estudio se aplica debido a la importancia de debatir este tema, y posibilitando así una reflexión crítica sobre los métodos de educación en salud. El objetivo fue reflexionar sobre las medidas profilácticas 
relacionadas con eventos adversos en Unidades de Cuidados Intensivos en pacientes ancianos, relacionándose con la importancia del proceso de educación en salud. Se trata de una revisión narrativa de la literatura, con un aspecto cualitativo. La búsqueda de datos se realizó en las siguientes bases de datos: Base de Datos de Enfermería (BDENF), Literatura Latinoamericana y del Caribe en Ciencias de la Salud (LILACS), Medline y Scientific Electronic Library Online (SciELO), identificadas a través de la Biblioteca Virtual en Salud (BVS). ) y Pub Med. La investigación se desarrolló de noviembre de 2021 al 15 de enero de 2022, y se utilizaron los descriptores "Anciano", "Prevención”, "Eventos adversos", "Unidad de cuidados intensivos" y "Educación en salud" establecidos en el listado Descriptores de Ciencias de la Salud ( DEC). Se constató que la ocurrencia de eventos adversos está directamente relacionada con fallas en la atención y falta de conocimiento para realizar prácticas profilácticas específicas para cada complicación, siendo las principales relacionadas con medicamentos, infecciones y heridas. Se concluye que la producción de reflexiones sobre este tema es fundamental para despertar en los profesionales y en la comunidad la importancia de debatir cuestiones relacionadas con la educación en salud, y así mejorar la atención ofrecida a los ancianos en cuidados intensivos.

Palabras clave: Adulto mayor; Prevención; Eventos adversos; Unidad de Cuidados Intensivos; Educación para la salud.

\section{Introdução}

A população de indivíduos com idade superior a 60 aumentou significativamente no decorrer dos anos, na década de 1950 havia cerca de 205 milhões de idosos no mundo, vindo esse quantitativo a crescer para 810 milhões em 2012, e cerca de 2 bilhões como estimativa para os anos de 2050 em diante, e com o processo de senescência também surgir o aumento das taxas de doenças associadas a idade, como exemplo as de origem cardiovascular (Boeckxstaens \& De Graaf, 2011).

Com o aumento do processo de senescência, e as altas taxas de comorbidade, surge um dos principais problemas relacionados a saúde pública, implicando diretamente em cuidados relacionados a profilaxia de comorbidades e superlotação de leitos hospitalares, com enfoque nas Unidades de Cuidados Intensivos (UTI) (Crivelaro et al, 2018). Sendo em média 60\% dos leitos ocupados por indivíduos com idade superior a 60 anos, o que gera maiores gastos aos órgãos responsáveis, pois os números chegam a serem 7 vezes maiores, quando relacionados a indivíduos com idade superior a 75 anos, necessitando assim de cuidados efetivos de monitorização e educação em saúde (Pias et al, 2015).

O processo de educação em saúde em ambientes de cuidados intensivos, se caracteriza como ação fundamental no processo de cuidados, principalmente nos aspectos relacionados a profilaxia de eventos adversos (Figueiredo et al, 2010). Sendo o conhecimento teórico, cientifico e prático, fatores diretamente ligados as taxas de surgimentos de eventos inoportunos causadores de complicações, e por isso devem ser repassados e divididos com os demais membros da equipe, através de diálogos informações e grupos de debates (Ruivo et al, 2020).

No entanto, ainda que essa definição seja mais adequada, percebe-se que o processo de educação em saúde ainda se baseia em um modelo verticalizado, em que o detentor de um saber mais especifico fornece informações a outro profissional, sendo esse apenas um deposito de conhecimento, e não havendo a troca de saberes, e por consequência não há construção de saberes, e não há profilaxia de eventos adversos (De Araújo et al, 2019).

Mesmo com esses métodos de educação em saúde, e sendo assim os profissionais cientes dessas concepções, esse processo ainda tende a ser caracterizado como hegemônico, quando a ação é realizada de forma prescritiva, e sem considerar o igualitarismo entre todos os membros da equipe multiprofissional no atendimento ao paciente em UTI (Filter de Andrade \& Simão, 2020). Devendo o processo de educar ser presente em todas as ações de cuidado e ser exercido por todos os profissionais da unidade, refletindo diretamente na qualidade do cuidado ofertado (Figueira et al, 2012).

Portanto, emerge a relevância do estudo, que se aplica a importância de se debater essa temática, e assim possibilitar uma reflexão crítica acerca dos métodos de educação em saúde. Logo, objetivou-se refletir acerca das medidas profiláticas relacionadas a eventos adversos em Unidades de Tratamento Intensivo em pacientes idosos, relacionando com a importância do processo de educação em saúde. 


\section{Metodologia}

Trata-se de um estudo do tipo revisão narrativa da literatura, de aspecto teórico-reflexivo, de abordagem qualitativa e viés exploratório. Conforme Rother (2007), esse tipo de revisão possibilita aos leitores adquirir conhecimentos e atualizações acerca de determinada temática, e assim se caracterizam componente fundamental para a educação continuada.

A elaboração dessa pesquisa foi realizada com base nos dados captados através das bases e bancos de dados: Base de Dados em Enfermagem (BDENF), Literatura Latino-Americana e do Caribe em Ciências da Saúde (LILACS), Medline, $e$ Scientific Eletronic Library Online (SciELO), identificados através da Biblioteca Virtual em Saúde (BVS) e PubMed, usando os descritores "Idosos", "Prevenção", "Eventos adversos", "Unidade de Terapia Intensiva" e "Educação em saúde", selecionados nos Descritores em Ciências da Saúde (DeCS) e também traduzidos para língua inglesa "Elderly", "Prevention", "Adverse events", "Intensive Care Unit" e "Health Education".

A pesquisa ocorreu no período de novembro de 2021 a 15 de janeiro de 2022. Foram atribuídos os seguintes critérios de inclusão: artigos completos de acesso gratuito, capítulos de livros, teses de doutorado, dissertações de mestrado nos idiomas português, inglês e espanhol. Excluíram-se estudos ou pesquisas que não abordavam a temática do referido estudo, ou apresentavam seu conteúdo com acesso restrito ou pago. Foram utilizados o operador booleano "AND" para as buscas, com os descritores de forma junto e separado.

Por se tratar de um estudo de revisão, a pesquisa não foi submetida ao Comitê de Ética em Pesquisa (CEP), ademais, reafirma-se a garantia dos preceitos éticos em todas as etapas na elaboração do estudo.

\section{Resultados e Discussão}

Do total de 223 publicações, dentre artigos publicados em periódicos, teses, dissertações e capítulos de livros. Foram realizados a leitura dos resumos e organizado o quantitativo de 12 estudos que se caracterizaram viáveis através dos critérios de inclusão, sendo esses organizados no programa Excel versão 2019.

O processo de educação em saúde no ambiente intra-hospitalar se caracteriza como fator essencial para uma assistência mais segura e humanizada, na qual o conhecimento é continuamente renovado através do diálogo entre os membros da equipe multiprofissional. Conforme Souza et al. (2018), as ações educativas se caracterizam como fator crucial na profilaxia de eventos adversos, sendo a pesquisa realizada com 241 pacientes, as complicações ocorreram em 152 deles, sendo $26 \%$ acima de 60 anos.

Em idosos em tratamento intensivo, observou-se que os principais incidentes decorrentes de uma assistência não efetiva foram, erros de medicações, infecções em geral, mais com enfoque as oriundas dos sítios de inserção e a ocorrência de lesões por pressão, que em sua maioria são evitáveis (Garzella et al., 2021). Apoiando essa afirmativa, com a pesquisa realizada por Barbosa et al (2021), na qual aponta os erros de doses, não realização das mudanças de decúbito, omissão de doses de medicações, aprazamento incorreto, falhas em manutenção de dispositivos de nutrição e hidratação, sendo esses agravos comumente associados a falhas na assistência multiprofissional, sendo imprescindível o planejamento, organização e treinamento da equipe.

O processo de capacitação e orientação através da educação é fundamental para correção de erros, principalmente relacionadas a praticas rotineiras da unidade. Segundo Oliveira e Nunes (2014), a admissão dos pacientes idosos na UTI, se caracterizam como principal momento de conversa entre os membros da equipe e troca de informações e orientações, porem a mesma ação não se caracteriza como continua no decorrer da estadia, o que contribui para a ocorrência de eventos adversos.

A construção de uma educação em saúde efetiva, é fundamental pois possibilita uma melhor efetividade nas ações de orientação, construção de protocolos, planejamento de cuidados sistematizados e na aplicação de técnicas profiláticas. Na pesquisa de Cruz (2018), aponta que o surgimento das lesões por pressão em idosos, está diretamente ligado a falhas nas 
mudanças de decúbito, na desinformação dos horários e em qual técnica usar para aliviar a tensão nas proeminências ósseas do paciente, e a não notificação dos casos, situações que poderiam ser corrigidas com um processo de educação em saúde.

De acordo com a pesquisa de Aguiar et al (2012), para que a estruturação de uma troca de conhecimentos seja firmada, é necessário que ocorra mudanças desde a formação dos futuros profissionais, tornando assim, o auxilio e desenvolvimento de técnicas relacionadas aos cuidados intensivos, algo mais simples e de melhor compreensão por parte dos demais membros da equipe, pois os mesmos detêm de uma base de conhecimentos consolidada.

Ademais, como fatores que dificultam as práticas educativas, aponta-se as altas cargas horarias de trabalho, o que corrobora para um cuidado "robotizado" na qual os profissionais não encontram tempo para realizar as discussões de casos e assim propor ações efetivas nos combates as principais complicações oriundas de erros relacionados ao cuidado (Felix et al, 2014).

Como limitação para o desenvolvimento do estudo, aponta-se a vasta quantidade de artigos relacionados a temática, porém com pouco enfoque na relação que a educação em saúde exerce na prevenção de efeitos adversos e melhoria dos cuidados ofertados pelos profissionais e por consequência os cuidados obtidos pelos pacientes idosos em cuidados intensivos.

\section{Conclusão}

Portanto, as ações de educação em saúde desenvolvidas para gerar segurança, e assim minimizar a ocorrência de eventos adversos em pacientes idosos em cuidados intensivos, são fundamentais e presentes diretamente ligadas ao cuidado efetivo desempenhados pela equipe multiprofissional.

Nessa percepção, emerge-se a necessidade de uma maior gama de estudos de cunho teórico-reflexivo, para que se dissemine nos locais de formação profissional e intuições hospitalares, a necessidade que ocorra diariamente e habitualmente a troca de conhecimentos entre todos os profissionais da saúde.

A produção de reflexões acerca dessa temática é fundamental para despertar nos profissionais e comunidade, a importância de debater assuntos relacionados a educação em saúde como forma profilática de eventos adversos na UTI, para assim melhorar a assistência prestada a população idosa que se encontra nesse ambiente.

\section{Referências}

Aguiar, A. S. C. d., Mariano, M. R., Almeida, L. S., Cardoso, M. V. L. M. L., Pagliuca, L. M. F., \& Rebouças, C. B. d. A. (2012). Percepção do enfermeiro sobre promoção da saúde na Unidade de Terapia Intensiva. Revista da Escola de Enfermagem da USP, 46(2), 428-435. https://doi.org/10.1590/s008062342012000200022

Barbosa, I. E. B., Fonseca, A. R., De Andrade, E. N. M., Maklouf, D. C., Ribeiro, M. C. S., Rodrigues, A. J. P. d. S., Laborda, Y. T. C., Da Silva, V. D. B. L., Lira, F. C. d. F., \& Gomes, S. S. d. S. (2021). Segurança do paciente: principais eventos adversos na Unidade Terapia Intensiva. Revista Eletrônica Acervo Saúde, 13(2), Artigo e6454. https://doi.org/10.25248/reas.e6454.2021

Boeckxstaens, P., \& De Graaf, P. (2011). Atenção primaria aos idosos: documento de posição de Fórum Europel de Atenção Primária. Qualidade de atenção primária, 19(6), 369-389.

Crivelaro, N., Contrin, L. M., Beccaria, L. M., Frutuoso, I. S., Da Silveira, A. M. R., \& Werneck, A. L. (2018). Adhesion of nursing to the blood current infection protocol. Revista de Enfermagem UFPE on line, 12(9), 2361. https://doi.org/10.5205/1981-8963-v12i9a234886p2361-2367-2018

Cruz F.F., Gonçalves R. P Simone., Raimundo R., Amaral M. A, segurança do paciente na UTI: uma revisão da literatura. Revista Científica FacMais, Volume. XII, https://revistacientifica.facmais.com.br

De Araújo, W. A., Assunção, M. L. d. B., De Araújo, I. S., Temoteo, R. C. D. A., De Souza, E. C., Almeida, G. D. S., Carvalho, F. O., \& Feitosa, A. D. N. A. (2019). Educação em saúde na Estratégia Saúde da Família: contribuições práticas do enfermeiro. Enfermagem Brasil, 17(6), 645. https://doi.org/10.33233/eb.v17i6.2231

Felix, T. A., Ferreira, F. V., Oliveira, E. N., Eloia, S. C., Gomes, B. V., \& Eloia, S. M. C. (2014). Prática Da Humanização Na Visita Em Unidade De Terapia Intensiva. Revista Enfermagem Contemporânea, 3(2). https://doi.org/10.17267/2317-3378rec.v3i2.381

Figueira, M. C. e. S., Leite, T. M. C., \& Silva, E. M. (2012). Educação em saúde no trabalho de enfermeiras em Santarém do Pará, Brasil. Revista Brasileira de Enfermagem, 65(3), 414-419. https://doi.org/10.1590/s0034-71672012000300004 
Research, Society and Development, v. 11, n. 2, e56711225901, 2022

(CC BY 4.0) | ISSN 2525-3409 | DOI: http://dx.doi.org/10.33448/rsd-v11i2.25901

Figueiredo, M. F. S., Rodrigues-Neto, J. F., \& Leite, M. T. S. (2010). Modelos aplicados às atividades de educação em saúde. Revista Brasileira de Enfermagem, 63(1), 117-121. https://doi.org/10.1590/s0034-71672010000100019

Filter de Andrade, V., \& Simão, J. P. S. (2020). Análise da aprendizagem baseada em problemas na educação profissional em saúde. Educação, Ciência E Saúde, 7(2). https://doi.org/10.20438/ecs.v7i2.320

Garzella, P. B., Bueno, D., \& Heineck, I. (2021). Detecçção de eventos adversos de eventos adversos relacionados a medicamentos por meio das ferramentas Global Trigger Tool e Medication Safety Thermometer: uma revisão narrativa. Clinical \& Biomedical Research. https://doi.org/10.22491/2357-9730.107252

Oliveira, C. N., \& Nunes, E. D. C. A. (2014). Caring for family members in the ICU: challenges faced by nurses in the interpersonal praxis of user embracement. Texto \& Contexto - Enfermagem, 23(4), 954-963. https://doi.org/10.1590/0104-07072014003590013

Pias, C., Mascolo, N. D. P., Da Silva, E. R. R., Linch, G. F. d. C., \& De Souza, E. N. (2015). Complexidade da assistência em unidade de terapia intensiva: subsídios para dimensionamento de pessoal de enfermagem. Cogitare Enfermagem, 20(3). https://doi.org/10.5380/ce.v20i3.41083

Rother, E. T. (2007). Revisão sistemática X revisão narrativa. Acta Paulista de Enfermagem, 20(2), v—vi. https://doi.org/10.1590/s0103-21002007000200001

Ruivo, B. A. R. d. A., Bastos, J. P. C., Júnior, A. M. d. F., Silva, J. C. d. S., De Jesus, L. M., Brígida, G. V. S., Dos Santos, C. B., Silva, C. d. S., Pinheiro, E. P. d. S., \& Camboim, B. B. P. (2020). Assistência de enfermagem na segurança do paciente na UTI: uma revisão integrativa da literatura. Revista Eletrônica Acervo Enfermagem, 5, Artigo e5221. https://doi.org/10.25248/reaenf.e5221.2020

Souza, R. F. d., Alencar, I. G. M., \& Alves, A. D. S. (2018). Eventos adversos na unidade de terapia intensiva. Revista de Enfermagem UFPE on line, 12(1), 19. https://doi.org/10.5205/1981-8963-v12i1a25205p19-27-2018 